\title{
PSICOLINGÜÍSTICA, NEUROLINGÜÍSTICA, LOGOPEDIA Y LINGÜÍSTICA CLÍNICA: JUNTOS SÍ, PERO NO REVUELTOS
}

\author{
Elena Garayzábal Heinze \\ $M^{a}$ Pilar Otero Cabarcos ${ }^{1}$
}

\begin{abstract}
RESUMEN
Cuando los lingüistas especializados en alteraciones del lenguaje recurren a bibliografía relativa a este tema, se encuentran con el sorprendente descubrimiento de que no hay apenas nada concreto acerca del tema descrito desde la propia lingüística. Efectivamente, nos encontramos con descripciones que nos adentran más bien en el campo de la terapia del lenguaje (logopedia) o nos remiten a la Psicología del Lenguaje con datos exhaustivos de la adquisición y mecanismos internos de producción y comprensión de mensajes, cuando no nos encontramos referencias detalladas del lenguaje de pacientes con lesiones cerebrales (afasias). Evidentemente, todas estas descripciones son importantes para el lingüista interesado por los aspectos clínicos del lenguaje, pero ¿dónde empieza una disciplina y dónde acaba otra? ¿Hasta qué punto la lingüística clínica puede concebirse como una disciplina autónoma? ¿Cómo puede contribuir la lingüística en la Psicolingüística y la Neurolingüística?

Palabras clave: lingüística clínica, logopedia, neurolingüística, psicolingüística, interdisciplinaridad.
\end{abstract}

\begin{abstract}
When linguists specialized on language disturbances look for more information about these speech pathologies, they find that there is little or no information from Linguistics itself. Certainly, we find out descriptions that lead us to the speech therapy area or which forward us to psycholinguistic definitions that have to do with language acquisition, and internal mechanisms of both comprehension and production of utterances. Other times we obtain exhaustive descriptions from disturbed language of patients with brain damage (aphasias). Of course, all the descriptions are of capital importance for the linguist who is interested on clinical aspects of language; but, where are the limits for each study? Is Clinical Linguistics an only and autonomous discipline? How can Linguistics contribute in fields as Psycholinguistics and Neurolinguistics?

Key words: Clinical linguistics, speech therapy, neurolingustics, psycholinguistics, crossdisciplinarity.
\end{abstract}

Elena Garayzábal, Ph.D. Profesora de la Universidad Autónoma de Madrid. Área de trabajo: Lingüística General (esp. en lingüística clínica)

Pilar Otero. Profesora de Secundaria y becaria de la Universidad de Santiago de Compostela. Área de trabajo:

Lingüística General (esp. en lingüística clínica)

Recepción: 30-08-2004

Aceptación: 18-11-2004 


\title{
1. Introducción
}

\begin{abstract}
Linguists and biologists, along with researchers in the relevant branches of psychology and anthropology, can move beyond unproductive theoretical debate to a more collaborative, empirically focused and comparative research program aimed at uncovering both shared (homologous or analogous) and unique components of the faculty of language (Hauser, Chomsky, Fitch 2002)
\end{abstract}

Es conocida la división entre lingüística teórica y lingüística aplicada, división que atiende fundamentalmente al criterio de finalidad u orientación del conocimiento, tal y como defiende Fernández Pérez (1996). El objeto de estudio de ambas es el mismo: el estudio científico del lenguaje humano a partir de la creación de modelos que describan y expliquen las lenguas (lingüística teórico-descriptiva) o a partir de su aplicación con fines educativos, prácticos o clínicos que respondan a problemas específicos que se planteen en las diferentes lenguas (Cardona 1991).

El modo de trabajar de los dos ámbitos de la lingüística es igualmente bien distinto y se justifica a partir del modo en cómo surgen ambos campos, así:

\begin{abstract}
Mientras que en el campo de la lingüística teórica las disciplinas están establecidas por su objeto de estudio y sólo después se integran en el área por la finalidad, por la orientación, en el terreno de la lingüística aplicada los ámbitos van emergiendo conforme se plantean problemas materiales y necesidades de solución. El objeto de interés no es, pues, previo a la orientación, sino que resulta del objetivo, de la exigencia y necesidad de solución (Fernández Pérez 1996: 22).
\end{abstract}

A pesar de estas aparentes divergencias que parecen oponer más que aunar los esfuerzos de ambas lingüísticas, no debemos caer en la creencia errónea de la existencia de una batalla entre ambas.

La relevancia de la lingüística en su faceta aplicada es un hecho que se confirma día tras día con el florecimiento y la aparición de disciplinas cada vez más atomizadas que se preocupan de aspectos concretos de la lingüística²:

(...) El lenguaje es tan valioso para el individuo, tan crítico para el funcionamiento eficiente de las sociedades humanas y tan asombrosamente intrincado y profundo en su estructura, que no puede dejar de atraer poderosamente la atención (...), esa atención debe producir estudios que tengan importancia práctica (por ejemplo en la terapia del lenguaje, la educación, las técnicas de traducción y otros muchos campos 'aplicados') (Fowler 1978: 43).

\section{En palabras de Fernández Pérez (1996: 22),}

(...) Por esa razón, por derivar los intereses de las necesidades materiales que se plantean, la Lingüística Aplicada se tacha de heterogénea y diversa.

(...) La variedad en el seno de la Lingüística Aplicada (...) no ha sido impedimento en la realidad para edificar áreas de aplicación y conseguir alcances resolutivos sobre aquellos problemas materiales planteados por el lenguaje y las lenguas. De este modo, ámbitos como didáctica de lenguas, Traductología, Lingüística clínica, Planificación lingüística o Lingüística computacional, son áreas con estructura, entidad y logros suficientes en las aplicaciones lingüísticas como para concederles relevancia y significación.

\section{Como bien señala Ángel López,}

en lo que llevamos de siglo, el siglo de la lingüística, nuestra disciplina ha pasado de ser una ocupación más o menos diletante y generalista a constituir una profesión especializada, casi una técnica rigurosa 
y precisa. Pudiera creerse que el surgimiento de los lingüistas como profesionales reconocidos es una consecuencia del alzamiento de la Lingüística a la condición de ciencia independiente. Sin embargo, no hay tal. La Lingüística como ciencia es antigua (1997: 107-8).

De hecho, estamos familiarizados con palabras como: "sociolingüística", "lingüística computacional”, "psicolingüística", "neurolingüística”, todas ellas áreas de la Linguiística, que constituyen asignaturas específicas de la propia titulación que se ofrece en diversas Universidades del territorio nacional (Universidad Autónoma de Madrid, Universitat Autònoma de Barcelona, Universidad Complutense, Universidad de Cádiz, Universidad de León), además de impartirse en la titulación de Filología Española ${ }^{3}$.

La existencia de este amplio abanico de áreas aboga y apoya la perspectiva multidisciplinar de la lingüística, su complejidad y su importancia, toda vez que nos abre caminos que antes parecían estar vetados a esta disciplina o en los que ésta tenía una participación e importancia mínima. De este modo, la Asociación Internacional de Lingüística Aplicada señala las siguientes áreas en las que la lingüística se interesa y participa activamente ${ }^{4}$ :

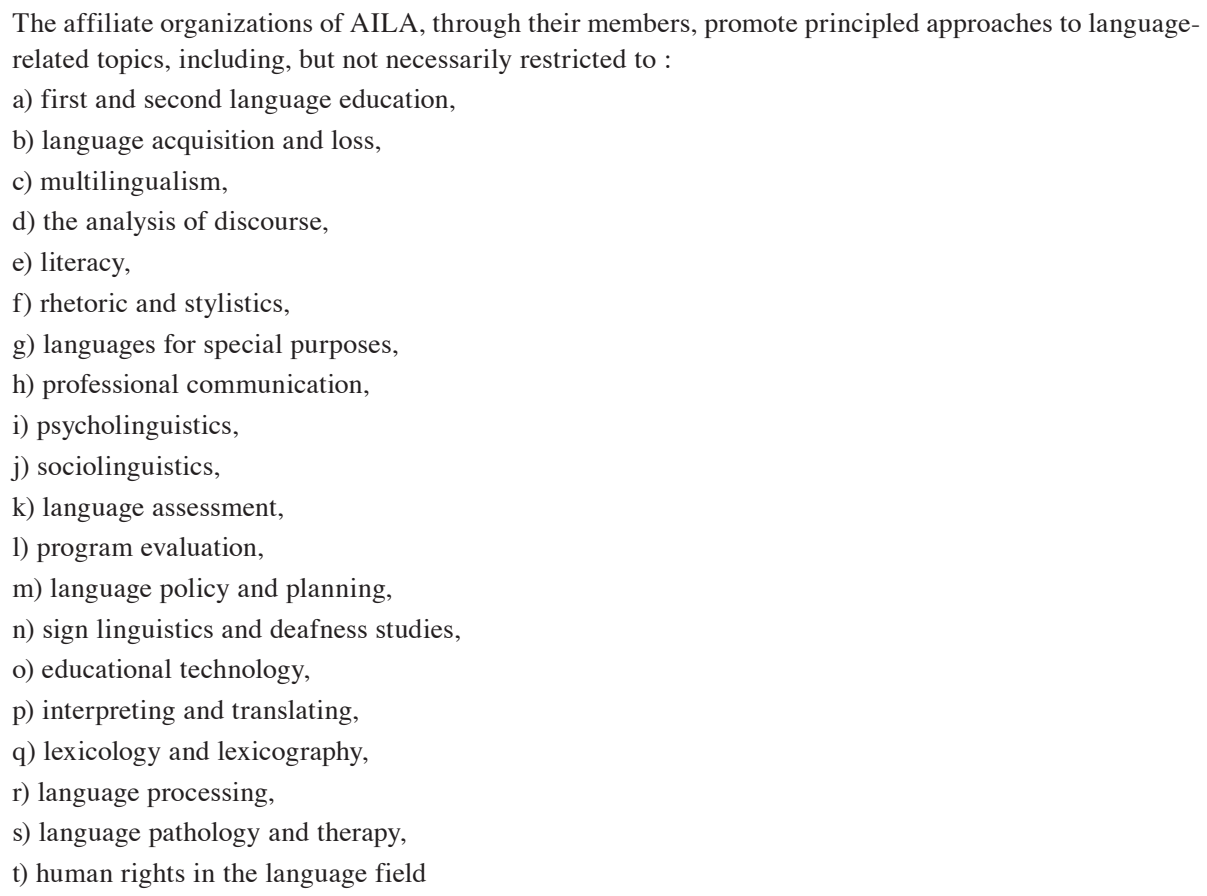

Así, desde hace relativamente poco tiempo, podemos encontrarnos dentro de las aplicaciones de la lingüística con una nueva disciplina de la lingüística; nos referimos a la lingüística clínica, cada vez menos desconocida, aunque cada vez más difusas sus fronteras entre la Psicolingüística, la Neurolingüística y la Logopedia, lo que lleva a pensar si tal vez constituya una nueva atomización de alguno o de todos estos campos. Tanto la Lingüística Clínica como la Psicolingüística y la Neurolingüística están "relacionadas entre sí por estudiar, cada una desde su posición, el lenguaje entendido como realidad neuropsicológica" (1999a: 200); aunque, en ocasiones, estas disciplinas no se han considerado dentro de la lingüística, tomándose incluso la interdisciplinariedad como una forma de intrusismo ${ }^{5}$. 
La indudable importancia de los modelos médicos o de los modelos psicológicos para conocer y tratar los trastornos comunicativos no puede, sin embargo, oscurecer o anular el papel que corresponde a los modelos lingüísticos en esta tarea. Conviene no olvidar que las deficiencias se reconocen como tales en los intercambios verbales y es en esta vertiente -en la que los trastornos se plasman- en donde interviene la lingüística (Fernández Pérez 2002: 9)

Desde estas páginas, intentaremos delimitar el campo de actuación de la lingüística, tanto en la Psicolingüística como en la Neurolingüística y la Logopedia, así como en un campo emergente como es la Lingüística Clínica, diferenciándola de las anteriores:

Uno de los retos a que se enfrenta la Lingüística en el tercer milenio es, sin duda, la sistematización de aquellos campos aplicados donde nuestra disciplina puede resultar una herramienta teórica importante (...) Existe un amplio campo de "otras aplicaciones" (diferentes a la traducción y la enseñanza de segundas lenguas) que, por lo general, carecen de construcción teórica propia y que existen tan sólo como prácticas concretas aisladas. (...) Tales prácticas son un objetivo legítimo para los estudios lingüísticos, y, más aún, un campo ineludible en la sociedad actual (Gallardo 2002: 129).

Una manifestación concreta de estas "prácticas aplicadas" sería la Lingüística Clínica, que se preocupa directamente de aspectos relacionados con las alteraciones del lenguaje, desde un punto de vista descriptivo, identificando y dando sentido a los datos observados tanto en niños como adultos,

al tiempo que (...) proporciona al terapeuta una base, un marco de referencia, un conjunto de principios sistemáticos que le permita actuar con mayor seguridad a la hora de organizar la información, evaluar el comportamiento lingüístico del sujeto, priorizar objetivos o diseñar estrategias de intervención (Codesido 1999: 197).

A pesar de que la lingüística en este ámbito de las alteraciones del lenguaje está aún poco desarrollada, podemos destacar algunos puntos en los que ya se ha avanzado, como nos recuerda Fernández Pérez (2002: 14): “el aspecto más sobresaliente ha sido el evidenciar que los componentes lingüísticos no se hallan mermados siempre de igual modo, y que en buena medida de los casos hay desequilibrios notables en el avance evolutivo de los componentes".

En definitiva, intentaremos poner cada disciplina en su lugar y ver cuáles son los lazos que las entrecruzan, relacionan y delimitan, reivindicando la importancia de la Lingüística, que desde la Psicología y la Neurología parece algo olvidada.

Por todos es sabido que en nuestro país el lugar del lingüista en este dominio ha sido muy modesto, ya que el grueso de la investigación descansa en manos de la Psicología y de la medicina. Pero la naturaleza de los problemas implicados en este terreno obliga, cada vez más a reivindicar el papel del lingüista como agente indiscutible del proceso (Codesido 1998).

Y, sin embargo, desde la Lingüística tenemos claro que:

La pertinencia de los campos de la Psicolingüística y de la Neurolingüística está fuera de toda duda en las coordenadas de la Lingüística actual, y no sólo por el interés de sus aportaciones en las esferas de formación señaladas -Logopedia o Lingüística en general- o en la extensión y profundización del conocimiento acerca del lenguaje, sino también por la incidencia de su papel en el diseño y elaboración de teorías de adquisición y de aprendizaje de la lengua, que, naturalmente, habrán de repercutir en las estrategias y técnicas de enseñanza (Fernández Pérez 1999: 88). 


\section{La Psicolingüística desde la Lingüística}

Si aceptamos que la lingüística es el estudio científico del funcionamiento del lenguaje $^{6}$ (Pinker 1994), que estudia su naturaleza y la capacidad que el ser humano tiene para él (Akmajian et al. 1984), que intenta igualmente ver y describir qué nos acerca y qué nos diferencia de otros sistemas de comunicación animal no humanos; y si partimos de la idea de que el hombre es un animal social, tal y como escribió Aristóteles, nos damos cuenta de que el objetivo principal de la lingüística es describir la competencia general propia y exclusiva del hombre, así como su actuación?

De esta manera, vemos que la actividad lingüística es un proceso de gran complejidad y cualquier disciplina que afronte su estudio inexcusablemente tendrá que abarcar campos temáticos y disciplinarios diferentes, no pudiéndose ignorar desde una disciplina los datos de otras disciplinas que tengan el lenguaje como tema principal de estudio. De hecho, la propia lingüística como disciplina abarca a otras en su búsqueda por explicar el lenguaje humano y las lenguas que han devenido.

Algunos planteamientos teóricos intentarán describir esta capacidad exclusiva desde una perspectiva universal (características comunes a todas las lenguas, universales lingüísticos) o desde una perspectiva particular (descripción de una lengua natural dada). Como consecuencia de estos planteamientos, surgen diversos enfoques metodológicos y prácticos; un ejemplo de ello serían los estudios interlingüísticos que parten de un área como la lingüística tipológica, cuyo afán puede ser muy ambicioso (búsqueda de universales por medio de la comparación de numerosas lenguas) o puede tener un objetivo más "humilde" cuando se limita a la comparación de unas pocas lenguas.

Los estudios interlingüísticos son importantes para la lingüística puesto que permiten comparar características estructurales de las diferentes lenguas del mundo, pero también permiten dilucidar si los procesos de desarrollo de estos principios estructurales son comunes en la adquisición de la lengua materna (L1) por parte del niño y si siguen un mismo desarrollo en niños que están en contacto y aprendiendo una segunda lengua (L2), como en el caso del bilingüismo; esto es, por una parte se ocupa de la comparación y descripción de lenguas naturales desde la perspectiva del lenguaje adulto (lingüística tipológica pura) y por otra, se ocupa de la comparación y descripción de las lenguas naturales desde la perspectiva de la adquisición y el desarrollo del lenguaje infantil tanto en situaciones típicas como atípicas. Este último aspecto ha sido tradicionalmente abarcado y estudiado por la Psicolingüística (psicólogos en su mayoría) y es precisamente en este punto donde el papel de los lingüistas se diluye.

La importancia de la lingüística aplicada tanto en el marco de la adquisición del lenguaje como de las alteraciones del habla y del lenguaje queda manifiesta en el hecho de que identifique, describa e interprete la conducta lingüística de una persona. Sin embargo, las aportaciones de otras disciplinas consideradas fuente de la lingüística han sido igualmente importantes, concretamente aquellas que toman como base la naturaleza neuropsicológica del lenguaje, esto es, la Psicolingüística y la Neurolingüística (Codesido 1999a).

La Psicolingüística, aunque fundamental, es un campo relativamente reciente ${ }^{8}$, pues los estudios lingüísticos anteriores a las primeras formulaciones de Chomsky sobre el innatismo sólo partían de la descripción del lenguaje adulto, y permitían a su vez la formulación de gramáticas normativo-prescriptivas, que daban la pauta de cómo el hablante debía hablar. El desarrollo de las teorías chomskianas acerca de la base innata del lenguaje, la pobreza 
de estímulos a la que está expuesto el niño, la existencia de un dispositivo de adquisición del lenguaje (DAL; LAD: Language Acquisition Device), llevan a describir los términos de competencia y actuación (vid. nota 3), que tienen su antecedente directo en los términos de langue y parole, formulados y descritos por Saussure (1916).

Todo lo anterior coincide con las teorías de Lenneberg (1964) acerca de la existencia de un periodo crítico para la puesta en marcha del lenguaje por parte del ser humano y que él estimaba en torno a los 10 años de edad, después de los cuales no se llega a adquirir una gramática completa de la lengua.

Estos datos son significativos ya que existe un antes y un después de las teorías de Chomsky, no sólo para la lingüística, que hasta entonces se centró en el estudio del lenguaje adulto y en la creación de modelos teóricos que permitieran explicar las lenguas del mundo, sino por lo que supone la aparición del enfoque psicolingüístico en el estudio del lenguaje y, por tanto, un interés específico en el lenguaje del niño, su adquisición y desarrollo, que permitió a su vez el nacimiento del interés por las alteraciones del lenguaje y sus repercusiones ${ }^{9}$.

En la Lingüística cartesiana (Chomsky 1966) se concibe el lenguaje como una propiedad natural de la mente humana, idea ésta que retoma los planteamientos de Descartes acerca de que el hombre, a diferencia de otros animales, posee facultades que no se pueden explicar de forma mecánica y por ello el lenguaje sería lo que expresara la diferencia entre la conducta del animal y la del hombre:

\footnotetext{
El hombre tiene una capacidad específica, un tipo único de organización intelectual que no puede atribuirse a órganos exteriores ni relacionarse con la inteligencia general y que se manifiesta en lo que podemos denominar aspecto creador del uso del lenguaje corriente, y cuya propiedad consiste en ser ilimitado en cuanto a su alcance y en no precisar estímulo (Chomsky 1966: 19).
}

Es, por tanto, un hecho indiscutible que la lingüística adopta un papel importante dentro de la Psicolingüística, interesándose, al igual que la Psicología, por el funcionamiento del lenguaje y el estudio de la mente; no olvidemos que, a partir de las teorías de Chomsky sobre el lenguaje, la lingüística se convierte, o reconvierte quizás, a una ciencia cognitiva: "El estudio de la lengua es parte de una empresa más general: representar con detalle la estructuración de la mente (...) una gramática es una estructura cognitiva que interacciona con otros sistemas de conocimiento y creencia" (Chomsky 1977: 47).

Según Radford (2000: 28):

La Psicolingüística trata la cuestión de cómo la gramática representada mentalmente (la competencia lingüística) es utilizada en la producción y comprensión del habla (la actuación lingüística). La forma más directa de enfocar esta relación es adoptar la hipótesis de que una gramática generativa puede ser estudiada simplemente como si ella misma diera cuenta de cómo comprendemos y producimos oraciones en tiempo real.

Si queremos definir la Psicolingüística, desde el punto de vista lingüístico, diremos, utilizando las palabras de Codesido (1999a: 198), que se trata de una disciplina teórica

\footnotetext{
caracterizada por presentar como objeto de estudio el lenguaje como fenómeno con una base psicológica mental. (...) sus principales intereses se localizan en torno a dos grandes temas: por un lado, la adquisición de la habilidad lingüística por parte del niño (...) y, por otro, la relación entre el comportamiento lingüístico y los procesos mentales de codificación y decodificación que subyacen a dicho comportamiento.

(...) La finalidad pasa por lograr conocimiento acerca de los procesos mentales que aparecen asociados al desarrollo de la habilidad del lenguaje y a la propia actividad lingüística en sí misma.
} 
Sin embargo, desde la óptica puramente psicológica, las concepciones cambian y se da un papel escaso o nulo a las aportaciones que la Linguiística pueda ofrecer. Así, la Psicolinguiística quedaría definida de la siguiente manera:

[la psicolingüística es] una rama de la psicología y tiene por objeto el uso del lenguaje, es decir de cómo utilizamos esos conocimientos que supuestamente todos poseemos sobre nuestro idioma materno y qué actividades mentales se ponen en juego al hablar y al escuchar, en la lectura y en la escritura (Valle 1991: 20).

Además, según este mismo autor, la función de la Lingüística consistiría en:

(...) la búsqueda, descripción y explicación, al nivel más formal y económico posible, de los elementos constituyentes de una lengua y de las relaciones de combinación entre ellos (...) En resumen se puede afirmar que la lingüística (...) trata de ver cómo se pueden formalizar estos distintos tipos de conocimientos lingüísticos en un sistema de reglas que capte las indudables regularidades que aparecen en la conducta (actuación) lingüística (...) (Ibid.: 19-20).

Por lo tanto, una de las principales relaciones entre la Lingüística y la Psicología, que a su vez parecen delimitarlas, sería que:

(...) La competencia sería el dominio propio de la lingüística en tanto que la actuación lo sería de la psicolingüística; tomados ambos términos en el sentido chomskiano de conocimiento tácito que posee un hablante ideal de las reglas capaces de generar todas las oraciones legales de un idioma y ninguna que no lo sea (...) (Valle 1991: 19).

Si tenemos en cuenta las opiniones de este autor, coincidentes con las de muchos otros psicólogos, está claro que el dicho popular de "zapatero a tus zapatos" relega a la Linguiística a su aspecto más teórico, no teniendo cabida en la práctica psicológica.

Sin embargo, no debemos olvidar el hecho de que no sólo los actuales modelos teóricos sobre el lenguaje que postulan los lingüistas tienen su base en la observación del lenguaje hablado, es decir, no son constructos teóricos que parten de la nada, sino que, de hecho, el lenguaje oral ya fue objeto de interés y de descripción por parte de los antiguos gramáticos clásicos en el siglo IV a.C. Por ello, podemos afirmar que la Lingüística no sólo estudia la competencia sino que se asienta sobre las bases de la actuación.

Este mismo autor incluso va más allá y no sólo defiende la distinción arriba indicada, sino que se inclina a una psicolingüística sin lingüística "si por tal se entiende una psicología del lenguaje cada vez más psicológica” (Valle 1991: 21).

Por otro lado, parece que para algunos psicólogos la Lingüística sólo ha tenido un único desarrollo: el referido a las gramáticas generativas que desarrolló Chomsky, uno de los lingüistas que más se conoce.

Desde la Lingüística, nos parece que hay una cierta confusión sobre algunos términos. ¿Es lo mismo Psicolinguiística que Psicología del lenguaje? No creemos que sea así. Como muestra de dicha confusión valgan las siguientes definiciones:

Desde la Psicología, se considera al psicolingüista como aquel "científico (normalmen$t e^{10}$ con formación psicológica) que estudia la forma en que las personas comprenden, producen o adquieren el lenguaje" (Pinker 1994).

Shula Chiat nos manifiesta que "los procesos psicolingüísticos (de producción) nos llevan de los significados de la mente a los movimientos motores de la boca" (2000: 11).

Por otro lado, Belinchón, Rivière e Igoa (1992: 62) nos proporcionan la siguiente caracterización: 
(...) a la psicología no le interesa el lenguaje como producto acabado o cerrado en sí mismo, lo que le interesa a la psicología del lenguaje es que éste, en sus diferentes formas, se adquiere, se usa y resulta funcional en sujetos y organismos de diferentes especies y entre ellos, y de forma muy particular, de la especie humana.

Desde la Lingüística, el lenguaje interesa dado que es un medio de comunicación preciso y complejo que sólo el hombre ha desarrollado y que le permite integrarse en la sociedad y establecer vínculos afectivos. No sólo se ha desarrollado como una forma de supervivencia, de la misma manera que en el resto de los seres vivos, quienes han desarrollado procesos básicos de comunicación con un fin puramente funcional: sobrevivir y aparearse. En el hombre, por el contrario, se han desarrollado mecanismos complejos desde el punto de vista físico u orgánico (desarrollando estructuras cerebrales y bucofonatorias concretas) y desde el punto de vista psicológico (la capacidad de planificación, de abstracción, razonamiento, etc.)

En su complejidad, el sistema de comunicación humano, por medio de un conjunto finito de sonidos articulados que constituyen el habla, es capaz de crear infinitos mensajes que los lingüistas podemos analizar desde diferentes puntos de vista: fonético-fonológico, gramatical, semántico y pragmático. Por este motivo, la idea clásica de que a los lingüistas nos interesa el lenguaje como producto acabado es errónea, dado que el lenguaje nos interesa sobre todo como sistema de comunicación, como herramienta que usamos diariamente en nuestras interacciones con los demás. Claro está que el lenguaje en uso tiene muy diferentes manifestaciones que dependen de múltiples variables (el hablante, el contexto, la situación) que son igualmente importantes a la hora de valorar las emisiones. Conforme a ello, desde las nuevas tendencias de la lingüística se sostiene que "las particularidades de desenvoltura lingüística propias de cada sujeto -incluso al margen de su trastorno y de su grado de limitación- exigen atender a su patrón comunicativo" (Fernández Pérez 2002: 16).

Si, como dice Chiat (2000), los psicolingüistas se centran en el proceso que va desde la mente hasta la manifestación externa del lenguaje, ¿quién analiza la manifestación externa? Claro está que una parte muy grande de este análisis se lleva a cabo desde la Lingüística, pero también desde la Psicología. Lo deseable sería que se tratase de psicólogos con una preparación lingüística de fondo. Esta aclaración es precisa, dado que es muy frecuente encontrar trabajos de psicolingüística realizados por psicólogos que nunca serían comprendidos por los lingüistas, dado que, entre otras cuestiones, la terminología que manejan no es la misma o nos resulta cuando menos confusa ${ }^{11}$.

La labor del lingüista resulta fundamental para la realización de una prolija descripción de los diferentes niveles de análisis lingüístico del hablante o grupo de hablantes, teniendo en cuenta tanto los diferentes niveles por separado como sus conexiones, toda vez que esto le permite crear teorías o suposiciones acerca del lenguaje y en un momento dado hacer frente a problemas concretos y darles una solución. En este sentido, se interesa tanto por el lenguaje que desarrollan los niños, como por el de los adultos, y tanto por los procesos de adquisición y aprendizaje, como por las alteraciones que pueda presentar.

Valga la opinión de Radford (2000: 18): 
que los datos con los que trabajan los lingüistas (proporcionados por ellos mismos o por otros hablantes nativos) normalmente tienen unas propiedades los suficientemente claras como para hacer innecesarios los experimentos controlados.

Asimismo, el estudio del desarrollo del lenguaje se ha convertido en una notable fuente de información para el lingüista, dado que los datos que se obtienen pueden aportar nuevas explicaciones para ciertas alteraciones e incluso podrían explicar algunos principios universales que subyacen a las lenguas (vid. Slobin). Aunque lo que nos resulta más interesante es que a través del estudio de tales datos se han llegado a reconocer particularidades reiterativas en las diferentes fases de adquisición. Y es que "no es tan importante comprobar la adecuación de la teoría como reconocer el progreso de la habilidad a través de códigos y perfiles lingüísticos y cognitivos en distintos estadios" (Fernández Pérez 2003: 277). Por ello, tal y como hemos mencionado más arriba, cada vez cobran más importancia los estudios interlingüísticos.

Contrariamente, el manejo de datos también nos ha servido para reconocer la heterogeneidad donde anteriormente sólo se consideraba un todo homogéneo. Convenimos con Fernández Pérez (2002: 14) en que:

\begin{abstract}
la consideración esencialmente lingüística de los niños con trastorno de lenguaje es relativamente reciente (...) la actitud descriptiva de las situaciones particulares en sus características individuales ha traído consigo la introducción de procedimientos analíticos de la Lingüística, de modo que los casos de "trastorno de desarrollo del lenguaje" han comenzado a contemplarse en su diversidad (...) El aspecto más sobresaliente ha sido el de evidenciar que los componentes lingüísticos no se hallan mermados siempre de igual modo, y que en buena parte de los casos hay desequilibrios notables en el avance evolutivo de los componentes.
\end{abstract}

Pues bien, parece que lo único que queda claro de este panorama tan confuso, es que para algunos psicólogos las aportaciones de la Lingüística son pecata minuta. Tampoco se toma en consideración que la Lingüística ha ido evolucionando y que no sólo se ha centrado en la competencia o el conocimiento implícito del supuesto hablante nativo ideal, sino que también se ha centrado en la actuación del hablante ideal y "no tan ideal" en situaciones de interacción, para lo que se ha fijado, además de en los aspectos más formales e internos de las lenguas, en los aspectos pragmáticos, extralingüísticos y sociales que le permiten su interacción con el mundo y con los demás; es decir, se interesa por el uso de la lengua en particular y del lenguaje en general, si bien es cierto que este interés ha sido más tardío que el de la Psicología ${ }^{12}$. Igualmente, la Lingüística se ha preocupado y ha contribuido considerablemente a la comprensión de cómo se originan los trastornos linguiísticos y de cómo puede rehabilitarse a los pacientes para superarlos. Estos aspectos, y por supuesto el hecho de que a los lingüistas les interese no sólo la competencia lingüística, han hecho que el punto de mira se dirija también a la actuación lingüística del niño y del adulto, tanto en situaciones típicas como patológicas. Este último foco de interés ha permitido el desarrollo de una nueva disciplina que es la Lingüística Clínica:

La lingüística aplicada, precisamente por su orientación resolutiva y el consecuente decantamiento hacia problemas existentes en las lenguas, ha de ocuparse de los fenómenos lingüísticos en su realidad y en todas sus dimensiones. (...) esa orientación resolutiva conduce a que la lingüística aplicada se centre en situaciones de déficits comunicativos producto de alguna patología (orgánica o funcional) con el propósito de perfilar la terapéutica apropiada que pueda corregirlos y paliarlos, tarea ésta que determina la emergencia de la lingüística clínica (Fernández Pérez 1996: 40-1).

Finalizamos este apartado revisando la descripción que, desde la lingüística, siguiendo a Cardona (1991: 230), haríamos de la Psicolingüística: 
(La Psicolingüística es la) rama de la Lingüística que estudia el comportamiento lingüístico desde el punto de vista psicológico y psicopatológico. Objeto de la psicolingüística son, entre otros, la adquisición del lenguaje, la relación entre lenguaje y centros motores, el lenguaje patológico, etc..

Y la Psicología del Lenguaje se define como "el estudio de temas que coinciden sustancialmente con los estudios de la psicolingüística, pero con mayor énfasis en los métodos psicológicos que en los lingüísticos" (Cardona 1991: 230).

Como se desprende de argumentos anteriores, defendemos que la Psicolingüística es una rama a caballo entre la Psicología y la Lingüística, en la que ambas tienen mucho que aportar. La situación ideal sería que hubiese un reconocimiento mutuo de las contribuciones que cada disciplina puede y debe aportar. En el fondo se trata de hacer efectiva la "interdisciplinariedad" de la que tanto se habla actualmente. "El problema de la complejidad de las materias relativas al lenguaje no es, en el fondo, un problema estricto de modelos, es un problema de situar cada cosa en su lugar. Es un problema de equilibrios, de flexibilidad y de apertura" (Peña-Casanova 1988: IX) .

\title{
3. La Neurolingüística desde la Lingüística
}

Tal vez las relaciones entre la Lingüística y la Neurología sean menos controvertidas y las diferencias entre ambas disciplinas, más claras; no obstante, como bien señala Codesido (1999a), el estudio y la intervención en las alteraciones del lenguaje ha estado siempre en manos de la Psicología y la Medicina y aún sigue estándolo, aunque es cierto que cada vez menos, como veremos en el siguiente apartado. Sin embargo, al contrario de lo que parece ocurrir con la Psicología, en el caso de la Lingüística y la Neurología parece que ambos campos tienen sus parcelas muy diferenciadas, aunque ambas se complementan mutuamente y se aportan datos relevantes.

Cuando entramos en el campo de la Neurolingüística nos movemos en torno a los datos proporcionados mayoritariamente por las observaciones del lenguaje patológico, pues "son los procesos patológicos los que provocan las disociaciones (en el sistema del lenguaje) que resaltan la arquitectura funcional del sistema del lenguaje - y proporcionan indicios referentes a los dispositivos orgánicos que son la base de esta arquitectura" (Rondal 1993: 15). Esto lo diferencia básicamente de la Psicolingüística, que habitualmente estudia el procesamiento del lenguaje en personas con desarrollo típico.

Jenkins (2000: 105) deja muy claro el papel de la Neurología:

\begin{abstract}
(...) Observamos que el estudio de la lingüística no nos dice necesariamente nada respecto a estos sorprendentes tipos de modularidad neuroanatómica. Nada en la GU (Gramática de universales) ni en la pragmática nos dice que el área de Broca está situada normalmente por encima y frente al oído izquierdo y el área de Wernicke se sitúa por encima de y detrás del oído izquierdo. Y tampoco puede predecir en qué parte del córtex acabarán situados el kanji japonés o el alemán como segunda lengua. Ni que el hemisferio izquierdo es donde se albergan fundamentalmente el lenguaje hablado y de signos. Los estudios de afasia, la proyección mediante estimulación eléctrica, la obtención de imágenes, etc., son formas apropiadas de investigar la modularidad anatómica, mientras que la pobreza de estímulo proporciona una prueba para estudiar las cuestiones de la modularidad de la gramática (una modularidad que puede en última instancia ser más evidente en la organización de los circuitos nerviosos que en la anatomía general). Por lo tanto, la GU y la neuropsicología proporcionan herramientas complementarias para arrojar luz sobre la organización del cerebro .
\end{abstract}

Entonces, ¿qué relación existe entre la Lingüística y la Neurología?, ¿qué estudia la Neurolingüística? 
La Neurolingüística es el estudio de cómo el cerebro hace posible el lenguaje. Valga una cita un tanto extensa que evidencia el interés común tanto de la lingüística como de la neurología por el lenguaje:

\footnotetext{
Los neurólogos estudian el cerebro y el sistema nervioso, pero aquellos que contribuyen al campo de la neurolingüística estudian la neurología humana y los trastornos del comportamiento cuando se ha producido alguna lesión en el cerebro o en el sistema nervioso (...) se preguntarían por el lugar preciso en el que se ha producido la lesión. La lingüística estudia el modo en que se estructura el lenguaje humano, pero aquellos lingüistas que han contribuido al campo de la neurolingüística centran su interés en la manera en que las estructuras del lenguaje pueden manifestarse en el cerebro (Obler et al. 2000).
}

Esto es, la Neurolingüística es un campo científico que se interesa por conocer los procesos y las estructuras que subyacen a la conducta verbal humana, con un especial interés en la alteración patológica de la conducta lingüística. Como bien señala Fernández Pérez (1992: 372), la Neurolingüística está particularmente interesada por "los procesos cerebrales implicados en la actividad lingüística".

En modo cómico, Hauser se plantea la siguiente cuestión:

\begin{abstract}
A neuroscientist might ask: What components of the human nervous system are recruited in the use of language in its broadest sense? Because any aspect of cognition appears to be, at least in principle, accessible to language, the broadest answer to this question isy, probably, "most of it". Even aspects of emotion and cognition not readily verbalized may be influenced by linguistically based thought processes (Hauser et al. 2002: 1570).
\end{abstract}

La lingüística, sus nociones y constructos teóricos, ha sido muy útil para el estudio de pacientes con lesiones cerebrales; pero no sólo eso, sino que algunas teorías lingüísticas, en su persecución de la gramática ideal y de los conocimientos que los hablantes tienen de su lengua, han recurrido necesariamente a contrastar datos de la competencia y de la actuación. En este último aspecto, la lingüística ha desarrollado nuevos campos de investigación que inciden principalmente en el estudio de la actuación para explicar la competencia y no al revés. Estos esfuerzos han sido fuertemente defendidos por la lingüística aplicada. De este modo, se defiende la idea de que "la emergencia de disciplinas como la Neurolingüística y la Afasiología Lingüística, con miras teóricas, y de la Lingüística Clínica, con pretensiones aplicadas, resulta inevitable y natural con nuevos planteamientos y orientaciones" (Fernández Pérez 1998).

Ya en 1969 se acuñó el término de Neurolingüística desde un foro dedicado a la lingüística aplicada. Hecaen y Dubois presentaron esta nueva aplicación como el análisis de las alteraciones del lenguaje debidas a causas neurológicas, con el propósito de lograr una clasificación de la afasia que tuviera en cuenta las zonas lesionadas del cerebro y una serie de conceptos lingüísticos.

El mapa cerebral que compete al lenguaje, aunque aún lejos de completarse, está siendo cada vez más definido: se sabe qué áreas y lóbulos cerebrales se relacionan mayoritariamente con el lenguaje (ya en su forma oral o escrita) y qué áreas tienen importancia fundamental en los diferentes niveles de análisis lingüístico, por poner algún ejemplo.

En este sentido, nos acercaríamos a la idea de Chomsky, quien considera la facultad de lenguaje como un órgano mental parecido a un órgano físico más de nuestra naturaleza. De alguna manera, esto supone considerar el cerebro como un órgano compuesto de diversos subcomponentes que se especializan en diferentes funciones como la visión, el lenguaje, la memoria. Dentro de la propia facultad del lenguaje se encontrarían propiedades distintas. 
Por otro lado, ante un daño cerebral puede ocurrir que algunos de estos subcomponentes se alteren de forma selectiva y queden afectados diversos aspectos lingüísticos aunque no de forma clara, dado que el cerebro no parece funcionar por áreas, sino que funciona y se interrelaciona de manera holística y una lesión puede tener consecuencias múltiples.

La Neurolingüística avanza a pasos agigantados y confluyen en este campo investigadores de muchas áreas, no sólo la Lingüística y la Neurología, sino también de la Logopedia y la Psicología.

Los neurólogos se han beneficiado de las nuevas corrientes y aplicaciones de la Lingüística que han aparecido con fuerza. Gracias a ésta, se han empezado a tener en cuenta problemas que anteriormente no se consideraban en personas que aparentemente no poseen problemas de lenguaje, o, como señala Obler (2000), se aplican actualmente pruebas a personas con alteraciones de la actuación para establecer si mantienen o no su competencia lingüística. Es decir, se va más allá de los trastornos del lenguaje, y esto se manifiesta también en un creciente número de trabajos en los que el bilingüismo es parte importante.

\section{Lingüística Clínica y Logopedia}

A muchos les sorprendería ver unidos en un mismo título Lingüística y Logopedia; y es que, desgraciadamente, aún se tiene una idea de la Lingüística que en poco se corresponde con la realidad. Algunos creen todavía que la Lingüística es una disciplina normativa y academicista que sirve para decir lo que está bien o no en una lengua, tal y como denuncia el Prof. Teixeira: “(...) o conceito que há sobre o que é a Linguiística: é o que diz o que está certo ou errado; é a policía da língua; é quem decide o que é que é linguisticamente correcto ou incorrecto (...)" (2002: 247).

El papel de los revisores ortográficos o estilísticos en la actualidad lo resuelven casi perfectamente algunos programas de ordenador, así que estimamos que ha llegado el momento de olvidar esa concepción propia de siglos pasados en los que la Gramática-y por ende la Lingüística - se ocupaba de este tipo de menesteres y enfrentarnos con el presente, cuando, desde nuestro punto de vista, ya no hay lugar para este tipo de "Lingüística". Se debe superar esa etapa en la que la figura del lingüista estaba indefectiblemente asociada a la del "magister":

\footnotetext{
É este papel de revisor ortográfico oficial que preenche praticamente na globalidade a imagem social (e académica) feita sobre os linguistas. É este papel, quase em exclusivo, de polícia-talibã para o combate ao vício e defesa da virtude (virtudes e vícios linguísticos, claro...) que, penso, nos devemos recusar a desempenhar como vocação prioritaria. Isto dá do lingüista a imagem do magister dixit medieval: a sua "ciência" consiste em saber muita coisa sobre o como-deveria-ser em vez de explicar o porquê do como-é (Teixeira 2002: 253).
}

Y tendremos que hacerlo desde la propia Lingüística, con investigaciones más centradas en la descripción de los datos, con una orientación más aplicada de estas mismas investigaciones; en fin, abriéndonos a un presente en el que prima lo resolutivo, en el que el mercado demanda soluciones a problemas reales; dando paso a una lingüística "joven".

Si bien es cierto que en las últimas décadas, gracias en parte-por qué no reconocerlo- a la ayuda de las Ciencias Cognitivas, se realzó el papel del lenguaje en todo lo que tiene que ver con los mecanismos cognitivos del ser humano, ello no provocó, como parecería lógico pensar, una revalorización del lingüista en el estudio de fenómenos que también son 
objeto de estudio de la Psicología, la Neurología, etc., sino que lo que suele suceder es que nos encontremos con un foniatra, un psicólogo o un informático que sea autodidacta en cuestiones relacionadas con la Lingüística.

Tendremos que romper con este hecho que ya empieza a ser tradición en nuestro país y convencer a la sociedad y al resto de profesionales, primero, de que la Lingüística es una ciencia, y después, de la necesidad de compartir conocimiento, de tener que dialogar con los lingüistas cuando se trate de solventar problemas, por ejemplo, como el que nos ocupa: las patologías del lenguaje. Somos conscientes de que hay todavía mucho campo que conquistar, muchos ámbitos en los que la Lingüística tendrá que hacerse un hueco para participar.

Si partimos de la sentencia de R. Jakobson, "La Lingüística es la ciencia de todas las manifestaciones del lenguaje", no dudaremos, primero, de que la lingüística sea o no una ciencia, y segundo, de que se deba ocupar o no de las patologías del lenguaje, ya que dichas patologías son una manifestación más del lenguaje. Pero, de nuevo en palabras del profesor Teixeira: “(...) Se a Lingüística não fizer passar uma imagem o mais correcta possível do que faz e do que pretende fazer, terá dificuldade em ocupar o lugar a que tem direito nesse mesmo mundo da ciência e da investigação" (Teixeira 2002: 248).

Esto es precisamente lo que pretendemos aclarar en este apartado: ¿qué es la Lingüística Clínica?, ¿de qué se encarga?, ¿qué hace o qué pretende hacer?

Parece difícil afrontar una sección como la que nos ocupa, sobre todo si tenemos en cuenta el objetivo último de este trabajo: dilucidar la diferencia entre Lingüística Clínica ${ }^{13}$, Psicolingüística y Logopedia, establecer fronteras entre estos campos del saber, cuando el objeto de estudio es tan cercano - incluso el mismo-, cuando el propio concepto de Logopedia varía de un país a otro (pues no se trata sólo de un cambio de etiqueta) ${ }^{14}$, y cuando en ocasiones los quehaceres de los profesionales se intercalan hasta diluirse.

Hay que tener en cuenta que la Lingüística Clínica es una área de conocimiento, una parcela de estudio, pero lo más importante es que los lingüistas somos seres humanos interesados en esta área y en otras, capaces también de atender otros extremos. Por eso somos los mismos lingüistas los que nos damos cuenta de la necesidad de contar con estudios de adquisición para poder evaluar una patología. Sin datos sobre la "norma", entendida como descripción y no prescripción, es decir, como lo habitual, no podremos comparar la patología con esa norma, no podremos desarrollar nuestro trabajo. Este es el motivo por el que a los lingüistas clínicos nos toque hacer también las veces de psicolingüistas e interesarnos por la adquisición y el desarrollo del lenguaje. Y este hecho se acentúa más en nuestro país, donde este trabajo todavía no está hecho, no es un campo cubierto, dado que apenas hay unos pocos trabajos centrados en la adquisición del español -y no hablemos ya del resto de lenguas peninsulares-, a diferencia de lo que ocurre con el inglés.

Por otra parte, la delimitación entre la Lingüística Clínica y la Logopedia, por su cercanía, por tratarse de disciplinas que estudian el mismo objeto, entraña en sí dificultades para establecer fronteras nítidas: "Clinical linguistics is the application of the theories, methods and findings of linguistics (including phonetics) to the study of those situations where language handicaps are diagnosed and treated" (Crystal 1984: 30).

Como ya señalaba Fernández Pérez en su artículo del año 1992, una situación como esta la encontramos en muchos otros campos del saber, puesto que 
al objeto particular de interés en cada caso (...) y el reconocimiento de la variedad de áreas pasa por la aceptación previa de la multiplicidad de enfoques y factores que el objeto admite por ser complejo (1992: 368).

Aunque con estas palabras la autora hacía referencia a la importancia de la distinción entre subdisciplinas, podemos extrapolarlas a la distinción entre dos ámbitos que comparten un mismo objeto de estudio, pues no existe objeto más complejo que el lenguaje. Ello no quiere decir que la distinción entre disciplinas que comparten un mismo objeto resulte imposible o inútil. Precisamos establecer fronteras entre disciplinas para alcanzar conocimientos más profundos sobre un mismo objeto de investigación.

Para hacer frente a dicha labor, empecemos por cuestiones que parecen tan elementales como saber qué significan las etiquetas "Lingüística Clínica" y "Logopedia". Seguimos, pues, las indicaciones de D. Crystal, para quien el etiquetaje (y su clarificación) era una de las primeras tareas que había que ejecutar.

Tratemos entonces de definir qué se entiende por Logopedia y qué por Linguiística Clínica en la actualidad. Para ello, qué mejor manera que acudir a la red.

A la pregunta, ¿qué es logopedia?, Lluisa Cabré responde de manera amplia: "el tratamiento de las perturbaciones del habla, del lenguaje y de la comunicación"15.

La respuesta se hace más específica en la página http://www.rediris.es/list/info/logopedia.es.html, pues se añade información sobre las distintas etiquetas que se utilizan para referirse a esta disciplina. "La logopedia es la disciplina que se ocupa del estudio y rehabilitación de estos trastornos, en diferentes países varía su denominación: 'SPEECH PATHOLOGY', 'SPEECH THERAPY', FONOAUDIOLOGÍA, ORTOFONÍA, ETC.” (http://www.rediris.es/list/info/logopedia.es.html, 13-05-04).

En la página de Néstor Pardo ${ }^{16}$, podemos encontrar un interesante artículo titulado "De la disciplinaridad a la transdisciplinaridad en Terapia del Lenguaje, Fonoaudiología o Logopedia", donde el autor define la Logopedia de manera tan ambigua que podría confundirse con la definición de Linguiística o de Psicología del Lenguaje:

La Terapia del Lenguaje, Fonoaudiología o Logopedia es la disciplina profesional de la Ciencia de la Comunicación Humana y sus Desórdenes, cuyo objeto de estudio es el proceso de interacción comunicativa del hombre, visto como el intercambio de mensajes en forma intencional y relevante entre dos o más participantes conscientes, y orientado a la aparición de una conducta o actitud cognitivo - comunicativa concertada que permita el acceso al conocimiento.

En la página web de la clínica FISIOMED de Salamanca ${ }^{17}$, definen de modo genérico la logopedia como la "ciencia que se encarga de la comunicación", pero más adelante precisa la logopeda Ana Belén Ramos Fernández:

La logopedia es la ciencia que se encarga de la terapia de la comunicación, por lo tanto el papel del logopeda es la responsabilidad de prevenir, evaluar y tratar los trastornos de la comunicación formada por el lenguaje, habla y voz. Estos trastornos son aquellos asociados a la comprensión y expresión del lenguaje tanto oral como escrito, y también de las formas de comunicación no verbales (http://www.clinica-fisiomed.com/p2h.htm, 13-05-04).

Contrariamente, en la página del gabinete online se nos ofrece una explicación que podemos cuando menos tildar de estrecha, al centrarse sólo en los problemas de habla: "rehabilitación de trastornos del habla (logopedia)", ya que indica una equivalencia entre ambos términos. 
En la misma línea se dirige la definición que nos ofrece el Diccionario de la Lengua

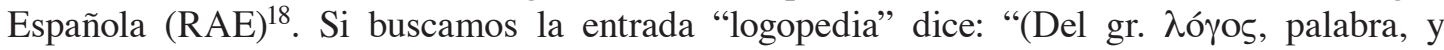
$\pi \alpha \iota \delta \varepsilon \iota$, educación). f. Conjunto de métodos para enseñar una fonación normal a quien tiene dificultades de pronunciación".

Asimismo, en la página http://www.iespana.es/logopeda, una profesional de Valencia define al logopeda como el "especialista en el tratamiento de los problemas del habla, malformaciones específicas de la boca y determinados retrasos, tanto en niños como en adultos" ${ }^{19}$.

En la página del Centro de Atención Temprana y Logopedia Díaz-Caneja ${ }^{20}$, nos ofrecen una interesante definición de Logopedia en la que la labor principal del logopeda se ceñiría a la intervención:

La logopedia, etimológicamente, significa enseñar las palabras. En la práctica, hace referencia a la intervención en las alteraciones en la comunicación, el lenguaje, el habla y la voz. (...) Por tanto, la Logopedia está dirigida a todas aquellas personas que presentan trastornos o dificultades en cualquiera de las mencionadas dimensiones del lenguaje, ya sean de tipo permanente o transitorio.

En www.espaciologopedico.com, en cambio, entienden que

\begin{abstract}
la logopedia tiene como finalidad: la prevención, el diagnóstico, el pronóstico, el tratamiento y la evaluación integral de los trastornos de la comunicación humana: ya sean éstos trastornos del habla o del lenguaje. El área de actuación de la logopedia y el ejercicio de la profesión se desarrolla en varios entornos: el educativo, el lingüístico, el conductual, el clínico, etc. (http://www.espaciologopedico.com/articulos2. asp?id_articulo=116, 13-05-04)
\end{abstract}

Observamos que la definición de Logopedia varía considerablemente dependiendo de la fuente que manejemos, tropezándonos tanto con descripciones detalladas como con panorámicas poco matizadas.

Desde nuestro punto de vista, la Logopedia trata diferentes alteraciones del habla, del lenguaje (oral y escrito) y de la comunicación en general (tanto los procesos de comprensión como los de producción) en niños y adultos ${ }^{21}$, y consideramos varios estadios en la práctica de la logopedia: prevención, diagnóstico, evaluación, asesoramiento, (...) hasta llegar a la intervención. Sobre todo, el trabajo de un logopeda consiste, entre otras cosas, en tratar toda esta serie de discapacidades comunicativas.

Pasemos ahora al terreno de la Lingüística Clínica: ésta aparece definida en la Enciclopedia del Lenguaje de D. Crystal (1994: 412) como un campo interdisciplinar basado en la "Aplicación de las teorías y métodos lingüísticos al análisis de los desórdenes del lenguaje hablado, escrito o sígnico". Y la define sobre todo como una subdisciplina aplicada:

\footnotetext{
Para mí, la lingüística clínica no es principalmente una nueva vía para resolver problemas en la teoría lingüística, pero, en primer lugar y sobre todo, una rama de la lingüística aplicada, un intento de utilizar la lingüística para resolver problemas en otros campos, como en logopedia o en enseñanza del lenguaje (Crystal 1986: 34).
}

Según este mismo autor, las contribuciones u objetivos específicos de la Lingüística Clínica se pueden resumir en ocho: (1) clarificación del metalenguaje; (2) descripción sistemática del comportamiento lingüístico del paciente, del terapeuta y de la interacción; (3) análisis de las descripciones con el fin de demostrar la extensión con la que los pacientes están utilizando sistemáticamente su lenguaje; (4) clasificación de los comportamientos lingüísticos de los pacientes, imprescindible para llegar a un diagnóstico diferencial; (5) evaluación de estos 
comportamientos a través de la comparación con las normas lingüísticas descriptivas válidas para el grupo en el que se enmarca el paciente; (6) formulación de hipótesis para la corrección del comportamiento lingüístico del paciente; (7) evaluación de los resultados de las hipótesis; y (8) evaluación de las estrategias correctivas utilizadas en la intervención, en tanto estén implicadas variables lingüísticas.

En cuanto a la aportación del lingüista en el ámbito clínico, estamos de acuerdo con la profesora Marrero cuando dice que los lingüistas tienen principalmente tres grados de participación, a saber:

\footnotetext{
I) Contribuir a la formación de los terapeutas del lenguaje; esto es, proporcionar a los estudiantes de Logopedia, Audición y Lenguaje, Audiología, Foniatría, etc. los fundamentos lingüísticos necesarios para el desarrollo de su trabajo.

II) Colaborar con los profesionales mencionados en tareas que requieran mayor especialización lingüística: el lingüista como experto para apoyo, consulta o cooperación.

III) Organizar y establecer una taxonomía de los trastornos del lenguaje desde un punto de vista lingüístico: la lingüística como eje estructurador del campo (Marrero 2000: 598-9).
}

Queda claro que aunque ambas disciplinas comparten un mismo objeto de estudio, las patologías del lenguaje, la distinción se hace obvia si nos fijamos en la metodología que utilizan y los objetivos que persiguen.

En el caso de la Lingüística Clínica, la metodología es más bien un modo de acercarse al problema. Casi podríamos hablar, parafraseando las palabras de Codesido (1999b), de una filosofía, donde importa, más que la etiología o los niveles de otras habilidades cognitivas (atención, memoria, inteligencia, conducta social, etc.), la descripción del problema (consiguiendo con ello un perfil lingüístico individualizado de cada paciente) y el análisis de todos los niveles del lenguaje (fonético-fonológico, morfológico, sintáctico y pragmático), dado que para describir sistemáticamente el lenguaje de un paciente hay que asegurarse de que todos los aspectos del lenguaje van a ser considerados. Y todo ello desde un enfoque no sólo interlingüístico sino también intralingüístico (por tanto, siempre se atiende a la norma del grupo en el que se enmarca el paciente).

La importancia de la labor del lingüista se observa claramente ante casos como el de una confusión de los sonidos [T] y [s], por poner un ejemplo, donde habrá que decidir primero si se trata de un mero rasgo dialectal o de un trastorno, y si es esto último, habrá que ver si se trata sólo de un déficit de pronunciación, afectando al plano fónico (que sería lo más palpable), o si además también nos encontramos con problemas en la concordancia, en cuyo caso también se encontraría afectado el nivel gramatical.

De otro lado, el objetivo de un lingüista nunca es la rehabilitación de un paciente. La terapia forma parte del trabajo del logopeda. Desde la Linguiística se trata de analizar, describir, para poder, eso sí, ayudar a solventar el problema. Digamos que el lingüista podría proporcionar herramientas con las que trabaje el logopeda. He ahí la trascendencia de la función orientadora del lingüista, y de la utilización de una metodología empírica, la única posible si lo que pretendemos es la aportación de datos, para poder prestar ayuda y asistencia en la rehabilitación de un paciente.

Entendemos que para corregir primero hay que evaluar, y para evaluar hay que analizar, y el análisis de algo presupone descripción. Por tanto, para llegar a la corrección, lo primero es la descripción. Los logopedas necesitan disponer de herramientas adecuadas para poder 
desarrollar su trabajo. En Gran Bretaña, por ejemplificar con uno de los países con más tradición en este ámbito, no se pone en duda el papel del lingüista en el campo de las patologías. Hace años que colaboran lingüistas, psicólogos y logopedas. El logopeda, responsable de la corrección, necesitará siempre una descripción y un análisis sistemático, cuyo encargado es el lingüista, dado que "las impresiones intuitivas no son suficientes si se quiere situar el campo de la patología del lenguaje sobre una base sólida, científica y que empiece a ser comparable a la medicina en cuanto a la confianza con la que hacen sus diagnósticos y pronósticos" (Crystal 1986: 42).

En este mismo sentido, versan las palabras escritas por el "maestro" M. Monfort en 1986, pero todavía actuales:

\begin{abstract}
Afortunadamente, el interés para con los trastornos del lenguaje demostrado por otras disciplinas, como la psicología y la lingüística (...), nos ha aportado a lo largo de estos últimos años, no sólo una cantidad de datos relacionados con nuestra materia y con los niños con los cuales trabajamos, sino también unas direcciones en las cuales poder orientar nuestro trabajo y aportar nuestra ayuda al esfuerzo común de comprensión de lo que ocurre en los trastornos de la comunicación y de lo que se puede hacer con seguridad para remediarlos o disminuir su impacto personal y social (Monfort 1986: 12).
\end{abstract}

El déficit lingüístico es un fenómeno interactivo porque es lenguaje. El lenguaje-y por ende sus trastornos-, por tratarse de un fenómeno de naturaleza social, no existe sin un interlocutor, sin el otro. Esto nos puede dar una idea de la importancia de la interacción; y no sólo nos estamos refiriendo al mecanismo de la comunicación, sino que esa importancia podemos extrapolarla a la interacción entre disciplinas, de manera que podemos afirmar que sin interacción no hay desarrollo de conocimiento. (...) É que, cada vez máis o mundo da ciência é composto por uma interligaçao de pesquisas e de intêr-
cambios (...). (Teixeira 2002: 248).

Nesta perspectiva, a Lingüística poderá ter uma relação privilegiada com a psicología, com a Neurología, com a Linguagem artificial (entre outras áreas do saber) (Teixeira 2002: 250).

Así, estamos convencidos de que la Lingüística sirve para algo más que para ser un refugio en sí misma de teorías que intentan explicar los distintos fenómenos lingüísticos, que la interdisciplinariedad es indispensable en el mundo científico.

\begin{abstract}
El cultivo de la Lingüística Clínica exige la colaboración con especialistas de otras áreas para disponer de la perspectiva integral y de la multidimensionalidad que exige el respeto por fenómenos tan complejos como los patológicos que -por padecerlos- necesitan paliativos y curación (Fernández Pérez 1998: 415).
\end{abstract}

Además, si nos quedamos con la dicotomía clásica ciencias versus letras, para nosotros "Lingüística" no tiene necesariamente una relación inmediata con "Letras". Nos interesa velar por la supervivencia (¿o quizás deberíamos decir por la génesis?) de la Lingüística como ciencia, con los mismos derechos que la Física o la Biología, por nombrar algunas de las ciencias tradicionales.

Cerramos recordando el ilustrativo lema que da título a este artículo: "juntos sí, pero no revueltos". Una de las principales pretensiones era delimitar fronteras y ello implica que hay varias disciplinas "en el ajo"; no debemos olvidarlo, la Lingüística es sólo una de ellas.

The goal of a theory of linguistic management is thus one to which linguistics can make an important contribution, but it is a limited one. It is limited by the need for the clinician to be aware of the whole range of psychological, social and of course medical factors which affect the well-being of his patient (...) Clinical linguistics may be one of the foundation-stones of speech pathology, but it is no more than that (Crystal 1981: 205). 


\section{Notas}

1. $\quad \mathrm{M}^{\mathrm{a}}$ Pilar Otero Cabarcos forma parte del grupo de investigación koiné [http://www.usc.es/koine], que desarrolla el proyecto de investigación "Elaboración de un test (lingüístico) para detectar déficit comunicativos en edad infantil”, el cual cuenta con el financiamiento del Ministerio de Ciencia y Tecnología (Plan Nacional de Investigación Científica, Desarrollo e Innovación Tecnológica, BFF2001-3234-C0201), con una aportación de un $70 \%$ de fondos FEDER.

2. No deja de tener su importancia el hecho de que el 11 de junio del 2002 tuviera lugar en la Universidad Complutense de Madrid una reunión profesional de lingüistas de todo el territorio nacional. Uno de los puntos principales de preocupación era concretar en el Boletín Oficial del Estado (B.O.E.) la presencia en otras titulaciones de la lingüística (Logopedia, Psicología, Informática se mencionaron expresamente). Esto da una idea bastante general de la situación de la Lingüística en nuestro país.

3. En la reunión de lingüistas que se celebró el 11 de junio del 2002 en la Universidad Complutense de Madrid, se manifestó la preocupación por el hecho de que en muchas Universidades las asignaturas que tienen el título de "lingüística" son impartidas por profesores que no son de Lingüística.

4. http://www.aila.soton.ac.uk/statutes02_01.htm,13-5-04

5. "Aunque las áreas de la Psicolingüística y de la Neurolingüística han estado escasamente atendidas en las Facultades de Letras y apenas se han hecho figurar en los Planes de Estudio -ya que, o bien se tomaban como materias de Medicina, caso de la Neurolingüística, o bien se concebían en un sentido exclusivamente conjetural y abstracto, caso de la Psicolingúística paralela a los planteamientos de la Gramática generativa-, este abandono no debe tomarse como indicador de la falta de interés y de relevancia de estas vertientes de formación Lingüística. Prueba de su importancia es su inclusión en el currículum de la Licenciatura en Lingüística, así como el reconocimiento del papel que les corresponde en esferas de Logopedia" (Fernández Pérez 1999: 73).

6. "Es un hecho que la mayoría de los manuales definen la lingüística como el estudio científico del lenguaje. Ninguna ciencia necesita justificar que lo es. Pero la tradición de las gramáticas académicas, así como las creencias populares sobre la lengua que todos hablamos, aconsejan insistir en la cientificidad de la lingüística para distanciarla de todos aquellos enfoques que no lo son. Ni la física, ni la química, ni las matemáticas, que son ciencias indiscutibles, pretenden cada una en su ámbito regular las conductas de nadie, como lo ha pretendido la gramática académica sobre el ámbito del lenguaje.

La lingüística procura actuar como las demás ciencias: mediante la observación rigurosa de los datos empíricos, trata de describir y explicar las estructuras que los organizan y las leyes que regulan dichas estructuras" (Martínez Celdrán 1995: 21)

7. Competencia y actuación son los conceptos tradicionales definidos por Noam Chomsky (1965). Por competencia entendemos el conocimiento implícito que el hablante tiene de su lengua por el simple hecho de usarla. Actuación sería la realización concreta en el habla de ese conocimiento implícito.

8. La utilización del término como tal ya fue acuñada en 1953, aunque el boom de la Psicolingüística queda reflejado en torno a los años 70 y 80 , coincidiendo con las teorías innatistas de Noam Chomsky que no sólo revolucionaron la Lingüística, sino también la Psicología.

9. "Las teorías lingüísticas que simbolizan la actividad verbal -todas ellas de raíz Psicolingüística o Neuropsicolingüística- tienen su exponente máximo en la gramática generativa, marco que jerarquiza 
los componentes de la lengua según su intervención en el proceso, si bien no determina si se trata de producción o de comprensión" (Fernández Pérez 2002:11).

10. El subrayado es nuestro. Como vemos, cabe la posibilidad, admitida por el propio psicólogo, de que los estudios de Psicolingüística no deben ser abanderados única y exclusivamente por los psicólogos.

11. Nos hacemos eco de las reflexiones de Fernández Pérez (1984), quien afirma que "el manejo de un mismo término en diferentes marcos teóricos conlleva cargas conceptuales diferentes en cada caso, pero no sólo eso, sino que si además sumamos la polivalencia significativa inherente al propio término, el resultado no es otro que un mar de ambigüedades que conducen a la pérdida del valor de la noción".

12. Desde la Lingüística, los aspectos pragmáticos comienzan a cobrar importancia en torno a los años 70, siempre coincidiendo con las teorías de Chomsky.

13. El término "Lingüística Clínica" es relativamente reciente, pues lo usa por primera vez David Crystal en su libro de 1981.

14. Así en España, Italia, Alemania, entre otros países europeos, hablamos de Logopedia, y se corresponde generalmente con estudios de diplomatura; en Francia, de Ortophonie; en Hispanoamérica, se habla de Fonoaudiología y se trata de una licenciatura; y en Estados Unidos se distingue entre SLP (Speech Language Pathology) y Audiología (orientada a la rehabilitación de sordos). "Pero hay una cosa que comparten todos los países: una falta de recursos financieros dedicados al estudio y tratamiento de los que probablemente son los trastornos humanos más olvidados de todos" (Crystal 1994: 264).

Para ampliar esta información acerca de los diferentes términos que se aplican al profesional que nosotros denominamos "logopeda", vid. artículo de Ana I. Codesido (1999: 43).

15. http://www.webpersonal.net/dav15405/lcabre/logpedi2.htm, 13-05-04

16. http://www.geocities.com/nestorpardo2000/transdisciplinarieta.html, 13-05-04

17. http://www.clinica-fisiomed.com/p3h.htm, 13-05-04

18. Diccionario de la Lengua Española (2001), s.v. "logopedia", Real Academia Española, Espasa-Calpe, S.A., Madrid, 22 ed.

19. http://www.iespana.es/logopeda/introduc.htm, 13-05-04

20. http://www.atencion-temprana.com/que_es/index.htm, 13-05-04

21. Tales como alteraciones miofuncionales, retraso del lenguaje, dislalia, fisura palatina, alteraciones de la voz, disartria, afasia, disfemia, dislexia, disortografía, disgrafía, discalculia, alteraciones de la memoria en edad avanzada o postraumáticas, alteraciones morfológicas de los pliegues vocales (nódulos, pólipos, edema de Reinke, etc.), parálisis laríngea, cambio de la voz, alteraciones auditivas, sordera, rh de implantados cocleares, la voz profesional (canto, locutores, actores, maestros, etc.), enfermedades degenerativas y demencias (Alzheimer, esclerosis, etc.), síndromes, estimulación precoz, fracaso escolar, técnicas de estudio, analfabetismo, sistemas aumentativos de comunicación, etc. 


\section{Bibliografía}

Akmajian, Adrian; Richard A. Demers y Robert M. Harnish. 1984. Lingüística: Una introducción al lenguaje y la comunicación. Madrid: Alianza.

Belinchón, Mercedes, Angel Rivière y José Manuel Igoa. 1992. Psicología del lenguaje. Investigación y teoría. Madrid: Trotta.

Cardona, Giorgio Raimondo. 1991. Diccionario de Lingüística. Barcelona: Ariel Lingüística.

Carbonero, Pedro, Manuel Casado y Pilar Gómez (eds.). 2000. Lengua y Discurso. Estudios dedicados al profesor Vidal Lamíquiz. Madrid: Arco Libros.

Casas Gómez, Miguel (dir.) y M.a Ángeles Torres Sánchez. (ed.). 1999. V Jornadas de Lingüística. Cádiz: Universidad de Cádiz.

Chiat, Shula. 2000. Los problemas de lenguaje en los niños. Madrid: Cambridge University Press.

Chomsky, Noam. 1970. Aspectos de la teoría de la sintaxis. Madrid: Aguilar (trad. española C.P. Otero).

1966. La lingüística cartesiana. Madrid: Gredos (Ed. española, 1972).

1977. Problemas actuales en teoría lingüística. Temas teóricos de gramática generativa. Madrid: Siglo XXI.

Codesido García, Ana Isabel. 1998. "Las discapacidades comunicativas en edad infantil: algunas implicaciones teóricas desde la Lingüística Clínica”. Revista de Logopedia, Foniatría, Audiología. 18 (4): 194-204.

1999a. "La Logopedia en Estados Unidos: estudios de postgrado". Revista de Logopedia, Foniatría, Audiología. 19 (1): 40-49.

1999b. "Aplicación clínica de la Lingüística: un ejemplo en la evaluación de la producción verbal espontánea en edad infantil”. R.I.L. (Revista de Investigación Lingüística). 2 (1): 43-58.

"Dos elefantes y una tela de araña (o de cómo lingüística y logopedia se balancean sobre la Malla Mundial)”. En: Luque Durán y Hernández (eds.), 165-177.

2002. "Acerca del Trastorno Específico del Desarrollo del Lenguaje (TEDL): revisión de algunos aspectos básicos y principales líneas de estudio". Actas del IV Congreso de Lingüística General. 2: 631-641. 
Crystal, David. 1972. "The case of linguistics: a prognosis". British Journal of Disorders of Communication. 7: 3-16.

1981. Clinical Linguistics. Londres: Edward Arnold.

1984. Linguistic encounters with language handicap. Londres: Edward Arnold.

1986. "Pasado, presente y futuro de la Lingüística Clínica”. En: Monfort (ed.), 34-42.

1994. Enciclopedia del Lenguaje. Madrid: Taurus (Ed. española J.C. Moreno Cabrera).

Crystal, David, Paul Fletcher y Michael Garman. 1989. Grammatical Analysis of Language Disability: a procedure for assessment and remediation. Londres: Edward Arnold ( $2^{\mathrm{a}}$ ed.).

De Molina, José Andrés y Juan de Dios Luque Durán (eds.). 1997. Estudios de Lingüística General. Granada: Método.

Duarte, Isabel Margarida (dir.). 2002. Actas do Encontro Comemorativo dos 25 anos do Centro de Lingüística da Universidade do Porto. Centro de Lingüística da Universidade do Porto.

Fernández González, Jesús et al. (eds.). 1999. Lingüística para el siglo XXI. I. Salamanca: Ediciones Universidad de Salamanca.

Fernández Pérez, Milagros. 1984. "El carácter de la ciencia lingüística”. Verba. 11: 129-156.

1992. "Consideraciones sobre el establecimiento y la demarcación de la Neurolingüística y la Psicolinguiística”. En: Martín Vide (ed.), 367-373.

1996. "El campo de la Lingüística Aplicada. Introducción”. En: Fernández Pérez (coord.), 11-45.

1998. "El papel de la teoría y de la aplicación en la construcción de disciplinas lingüísticas. El caso de la «Lingüística Clínica» y áreas conexas”. Revista Española de Lingüística. 28 (2): 389-419.

1999a. "El lingüista y las patologías del lenguaje". En: Fernández González et al. (eds.), 633-639. 
1999b. "La Psicolingüística y la Neurolingüística en el conjunto de la Lingüística". En: Casas Gómez (dir.) y Torres Sánchez. (ed.), 73-94.

2000. "Patologías del lenguaje y su reflejo psicosomático". En Hernández Sacristán y Veyrat Rigat (eds.), 81-100.

2002. "Importancia de los modelos lingüísticos en el estudio de los trastornos comunicativos". En: Hernández Sacristán y Serra Alegre (eds.), 9-25.

2003. "Dinamismo construccional en el lenguaje infantil y teoría lingüística". ELUA (Estudios de Lingüística Universidad de Alicante). 17: 273-287.

(coord.). 1996. Avances en Lingüística Aplicada. Colección “Avances en...”. 4. Santiago de Compostela: Universidad de Santiago de Compostela.

Fowler, Roger. 1974. Para comprender el lenguaje. Una introducción a la lingüística. México: Editorial Nueva Imagen (Ed. española, 1978).

Gallardo Paúls, Beatriz. 2002. "Fronteras disciplinarias: pragmática y patología del lenguaje”. En: Hernández Sacristán y Serra Alegre (coords.), 129-174.

Hauser, Marc D, Noam Chomsky y W. Tecumseh Fitch. 2002. "The faculty of language: What is it, who has it, and how did it evolve". Science. 298: 1569-1579.

Hecaen, Henry y Jean Dubois. 1971. “La neurolinguistique”. En: Perren y Trim (eds.), 85-99.

Hernández Sacristán, Carlos y Montserrat Veyrat Rigat (eds.). 2000. Lenguaje, Cuerpo y Cultura. València: Universitat de València.

Hernández Sacristán, Carlos y Enric Serra Alegre (eds.). 2002. Estudios de Lingüística Clínica. Valencia: Nau Llibres.

Iglesias Álvarez, Ana. 2002. "La observación como método de investigación empírica en Lingüística”. Verba. 29: 329-350.

Jenkins, Lyle. 2000. Biolingüística. Madrid: Cambridge University Press.

López García, Ángel. 1997. “La Lingüística del siglo XXI: ¿Hacia dónde se encamina la ciencia del lenguaje?”. En: De Molina y Luque Durán (eds.), 1, 107-132.

Luque Durán, Juan de Dios y Antonio Hernández. 2000. Trastornos del lenguaje: una perspectiva logopédica y lingüística. Granada: Método. 
Marrero Aguiar, Victoria. 2000. "La Lingüística Clínica". En: Carbonero, Casado y Gómez (eds.), 597-606.

Martín Vide, Carlos (ed.). 1992. Lenguajes naturales y lenguajes formales. Actas del VII Congreso de Lenguajes Naturales y Lenguajes Formales. Barcelona: P. P. U.

Martínez Celdrán, Eugenio. 1995. Bases para el estudio del lenguaje. Barcelona: Octaedro.

Monfort, Marc (ed.). 1986. Investigación y Logopedia. Madrid: CEPE.

Obler, Loraine K. y Kris Gjerlow. 2000. El lenguaje y el cerebro. Madrid: Cambridge University Press.

Peña Casanova, Jordi. 1998. Manual de Logopedia. Barcelona: Masson.

Perren, G. E. y John L. Trim (eds.). 1971. Applications of Linguistics. London: Cambridge University Press.

Pinker, Steven. 1994. El instinto del lenguaje. Madrid: Alianza (Ed. española, 1995).

Saussure, Ferdinand de. 1916. Curso de lingüística general. Buenos Aires: Losada (Ed. española, 1968).

Radford, Andrew et al. 2000. Introducción a la Lingüística. Madrid: Cambridge University Press.

Real Academia Española. 2001. Diccionario de la Lengua Española. Madrid: Espasa-Calpe (22 ed.).

Rondal, Jean. 1993. "Modularidad del lenguaje. Datos, teorías e implicaciones terapéuticas". Revista de Logopedia, Foniatría y Audiología. 23 (1): 14-22.

Teixeira, José. 2002. "Lingüística para poetas: o papel e as inter-relaçoes das Ciências da Linguagem perante as outras ciências". En: Duarte (comp.), 247-253.

Valle Arroyo, Francisco. 1991. Psicolingüística. Madrid: Morata. 